\title{
Identifying protein-protein interface via a novel multi-scale local sequence and structural representation
}

\author{
Fei Guo ${ }^{1 *+}$ (D), Quan Zou ${ }^{2+}$, Guang Yang ${ }^{3}$, Dan Wang ${ }^{4}$, Jijun Tang ${ }^{1,5}$ and Junhai $\mathrm{Xu}^{1+}$
}

From 14th International Symposium on Bioinformatics Research and Applications (ISBRA'18)

Beijing, China. 8-11 June 2018

\begin{abstract}
Background: Protein-protein interaction plays a key role in a multitude of biological processes, such as signal transduction, de novo drug design, immune responses, and enzymatic activities. Gaining insights of various binding abilities can deepen our understanding of the interaction. It is of great interest to understand how proteins in a complex interact with each other. Many efficient methods have been developed for identifying protein-protein interface.
\end{abstract}

Results: In this paper, we obtain the local information on protein-protein interface, through multi-scale local average block and hexagon structure construction. Given a pair of proteins, we use a trained support vector regression (SVR) model to select best configurations. On Benchmark v4.0, our method achieves average $I_{\text {rmsd }}$ value of $3.28 \AA$ and overall $F_{\text {nat }}$ value of $63 \%$, which improves upon $I_{\text {rmsd }}$ of $3.89 \AA$ and $F_{\text {nat }}$ of $49 \%$ for $Z R A N K$, and $I_{\text {rmsd }}$ of $3.99 \AA$ and $F_{\text {nat }}$ of $46 \%$ for ClusPro. On CAPRI targets, our method achieves average $I_{\text {rmsd }}$ value of $3.45 \AA$ and overall $F_{\text {nat }}$ value of $46 \%$, which improves upon $I_{\text {rmsd }}$ of $4.18 \AA$ and $F_{\text {nat }}$ of $40 \%$ for $Z R A N K$, and $I_{\text {rmsd }}$ of $5.12 \AA$ and $F_{\text {nat }}$ of $32 \%$ for ClusPro. The success rates by our method, FRODOCK 2.0, InterEvDock and SnapDock on Benchmark v4.0 are 41.5\%, 29.0\%, 29.4\% and $37.0 \%$, respectively.

Conclusion: Experiments show that our method performs better than some state-of-the-art methods, based on the prediction quality improved in terms of CAPRI evaluation criteria. All these results demonstrate that our method is a valuable technological tool for identifying protein-protein interface.

Keywords: Protein-protein interface, Multi-scale local average block, Hexagon structure construction

\section{Background}

In biological processes, many proteins carry out the special biological functions through protein-protein interactions, such as drug design and functional analysis. Gaining insights of various binding abilities can deepen our understanding on protein-protein interface. Determination of binding sites is widely applied in molecular biology research. It is of great interest to understand how

\footnotetext{
*Correspondence: fguo@tju.edu.cn

${ }^{\dagger}$ Fei Guo, Quan Zou and Junhai Xu are joint corresponding authors.

${ }^{1}$ College of Intelligence and Computing, Tianjin University, Tianjin, People's Republic of China

Full list of author information is available at the end of the article
}

proteins bind with each other, which helps us understand energetics and mechanisms of complexes. How to build more effective models based on sequence information, structure information and physicochemical characteristics, is the key technology for identifying protein-protein interface. There are many efficient techniques for the protein-protein interface prediction [1-11].

Some approaches use machine learning methods and statistical methods to analyze the differences between interface residues and non-interface residues on the surfaces [12-15]. ProMate [16] creates the circle around each surface residue, which can be used to extract the statistical histogram of many features. Then, it estimates the 
probability of each circle to be on the interface, and some circles with high probability values are clustered to identify binding residues. PPI-Pred [17] generates an interacting patch and a non-interacting patch for each training protein, and extract several features from these patches to build an SVM model for predicting the interacting patch in each testing protein. PINUP [18] proposes an empirical scoring function, including interface propensity and residue conservation score. It calculates the occurrence of each top scoring spot, therefore predicts residues on interface spots. Meta-servers combine the strengths of some existing approaches: meta-PPISP [19] combines three prediction servers; metaPPI [20] combines five identification methods. ProBiS $[21,22]$ predicts protein-protein interface by local structure alignment. It compares the information of a testing protein to some binding sites in the known database, for detecting similar structural residues.

Another kind of methods check the possible poses of two subunits; that is, how these subunits may dock. Docking methods based on fast Fourier transformation (FFT) [23], geometric surface matching [24], as well as intermolecular energy [25] have been proposed. The general approach is to explore all possible poses, and use one energy function to identify near-native poses. The problem of exploring all possible poses has been well-solved by some methods [26-28]. The key issue here is to design an energy function based on various properties and features that can identify near-native poses, such as hydrophobic and conserved polar at specific locations [29], hydrogen bonds and salt bridges [30], secondary structure composition [31], relative surface area burial and weighted hydrophobicity [32], force field energy evaluation [33-35]. FRODOCK 2.0 [36] presents an user-friendly proteinprotein docking server based on an improved version including a complementary knowledge-based potential. InterEvDock [37] is a server for protein docking based on a free rigid-body docking strategy, intergrating coevolutionary information. SnapDock [38] is a highly efficient template-based protein-protein docking algorithm, utilizing the interface PIFACE library. CIPS [39] proposes a new pair potential combining interface composition with residue-residue contact preference, screening docking solutions obtained either with all-atom or with coarsegrain rigid docking. ZRANK $[40,41]$ combines an atombased potential (IFACE) with five residue-based potentials for ranking solutions. It provides fast and accurate rescoring models from ZDOCK. ClusPro [42] develops a fast algorithm for filtering docked conformations with good surface complementarity and ranking them based on their clustering properties. RosettaDock [43] constructs the energy function by using van der Waals energies, orientation-dependent hydrogen bonding, implicit Gaussian solvation, side-chain rotamer probabilities and a low-weighted electrostatics energy. HADDOCK [44] makes use of the biochemical and biophysical interaction data, such as chemical shift perturbation data resulting from NMR titration experiments.

In this paper, we calculate the local information on the protein-protein interface, through multi-scale local average block and hexagon structure construction. Given a pair of input proteins, we use the trained support vector regression (SVR) model to select best protein-protein docking poses. Experiments show that our method achieves better results than some state-of-the-art methods. Here, we use the CAPRI evaluation criteria [45], $I_{\text {rms }}$ value and $F_{\text {nat }}$ value. On Benchmark v4.0 [46], our method has average $I_{\text {rmsd }}$ value of $3.28 \AA$ and overall $F_{\text {nat }}$ value of $63 \%$. On the CAPRI targets, our method has average $I_{r m s d}$ value of $3.45 \AA$ and overall $F_{\text {nat }}$ value of $46 \%$. The success rates by our method on Benchmark v4.0 are $41.5 \%$. Comparing to the existing methods, our method is a valuable technological tool for identifying protein-protein interface.

\section{Methods}

We find the relative orientation and position between two subunits, and each relative orientation and position combination is referred to as a configuration or pose. Given a configuration, we can determine the interface region between two subunits and fix the orientation as well as position of the regions far from the interface.

Here, we utilize our previous enumeration method [47] to identify the docking configurations of two subunits. It performs a large number of rigid transformations to enumerate the poses. Then, we design a novel energy function and build a trained SVR model to evaluate docking poses and select the top-ranking poses with lowest energy values. The flowchart is shown in Fig. 1.

In this paper, our main work is to obtain the local information on protein-protein interface for energy evaluation. First, each pair of proteins can be encoded with physicochemical property and position specific scoring matrix. Then, we establish two novel models, multi-scale local average block and hexagon structure construction, for representing local sequence and structural information on protein-protein interfaces. Finally, our proposed properties can be effectively applied to identify docking poses, as well as existing energy items.

\section{Physicochemical property}

We can use six physicochemical properties $[48,49]$ to extract protein features, since one protein can be represented by a vector of physicochemical property. These physicochemical properties are analyzed as hydrophobicity $(\mathrm{H})$, volumes of side chains of amino acids (VSC), polarity (P1), polarizability (P2), solvent-accessible surface area (SASA) and net charge index of side chains (NCISC) of amino acid, respectively. The physicochemical property 


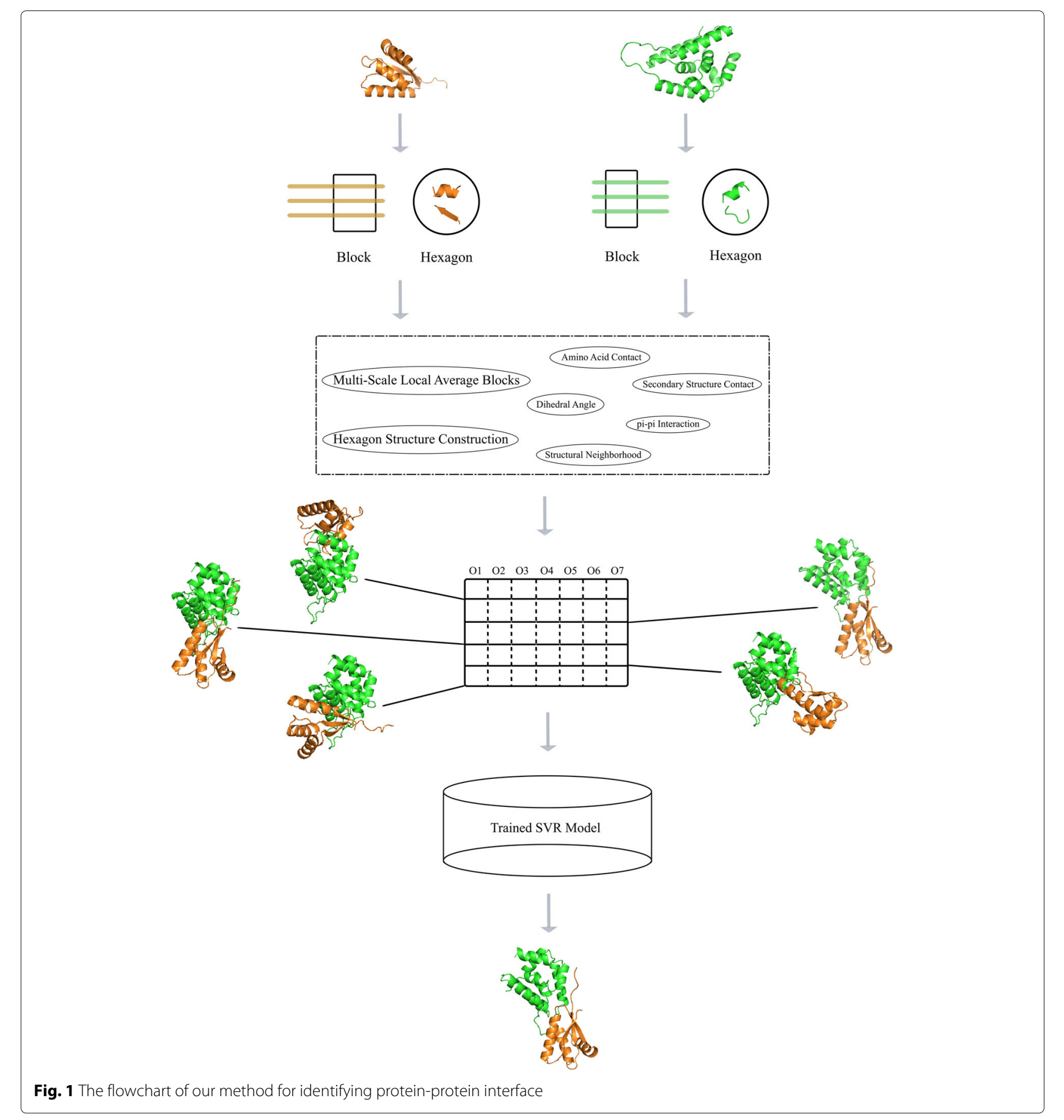

values of 20 amino acid types are shown in Table 1. They can be normalized to zero mean and unit standard deviation (SD) as follows:

$$
P_{i, j}^{\prime}=\frac{P_{i, j}-P_{j}}{S_{j}} ; \quad i=1,2, \ldots, 20 ; j=1,2, \ldots, 6
$$

where $P_{i, j}$ is the value of physicochemical property $j$ for amino acid type $i, P_{j}$ is the mean over 20 amino acid types of physicochemical property $j$, and $S_{j}$ is the corresponding standard deviation of physicochemical property $j$.

\section{Position specific scoring matrix}

The protein evolutionary information can be described by Position Specific Scoring Matrix (PSSM), generated by PSI-BLAST [50]. Given a protein, the PSSM information is stored in the $L \times 20$ matrix (protein length: $L$; amino acid types: 20 ), calculated as follows: 
Table 1 Original values of six physicochemical properties for 20 types of amino acids

\begin{tabular}{lllllll}
\hline Amino Acid & H & VSC & P1 & P2 & SASA & NCISC \\
\hline A & 0.62 & 27.5 & 8.1 & 0.046 & 1.181 & 0.007187 \\
C & 0.29 & 44.6 & 5.5 & 0.128 & 1.461 & -0.03661 \\
D & -0.9 & 40 & 13 & 0.105 & 1.587 & -0.02382 \\
E & -0.74 & 62 & 12.3 & 0.151 & 1.862 & 0.006802 \\
F & 1.19 & 115.5 & 5.2 & 0.29 & 2.228 & 0.037552 \\
G & 0.48 & 0 & 9 & 0 & 0.881 & 0.179052 \\
H & -0.4 & 79 & 10.4 & 0.23 & 2.025 & -0.01069 \\
I & 1.38 & 93.5 & 5.2 & 0.186 & 1.81 & 0.021631 \\
K & -1.5 & 100 & 11.3 & 0.219 & 2.258 & 0.017708 \\
L & 1.06 & 93.5 & 4.9 & 0.186 & 1.931 & 0.051672 \\
M & 0.64 & 94.1 & 5.7 & 0.221 & 2.034 & 0.002683 \\
N & -0.78 & 58.7 & 11.6 & 0.134 & 1.655 & 0.005392 \\
P & 0.12 & 41.9 & 8 & 0.131 & 1.468 & 0.239531 \\
Q & -0.85 & 80.7 & 10.5 & 0.18 & 1.932 & 0.049211 \\
R & -2.53 & 105 & 10.5 & 0.291 & 2.56 & 0.043587 \\
S & -0.18 & 29.3 & 9.2 & 0.062 & 1.298 & 0.004627 \\
T & -0.05 & 51.3 & 8.6 & 0.108 & 1.525 & 0.003352 \\
V & 1.08 & 71.5 & 5.9 & 0.14 & 1.645 & 0.057004 \\
W & 0.81 & 145.5 & 5.4 & 0.409 & 2.663 & 0.037977 \\
Y & 0.26 & 117.3 & 6.2 & 0.298 & 2.368 & 0.023599 \\
\hline & & & & & &
\end{tabular}

$\operatorname{PSSM}(i, j)=\sum_{k=1}^{20} \omega(i, k) \times D(k, j) ; \quad i=1, \ldots, L ; j=1, \ldots, 20$

where $\omega(i, k)$ is the frequency of amino acid type $k$ at the position $i$, and $D(k, j)$ is the value of Dayhoff's mutation matrix (substitution matrix) [51] between amino acid types of $k$ and $j$.

These PSSM elements can be normalized in a range of $[0,1]$ using the min-max normalization as follows:

$$
\begin{aligned}
\operatorname{PSSM}^{\prime}(i, j) & =\frac{\operatorname{PSSM}(i, j)-P S S M_{\text {min }}}{P S S M_{\text {max }}-P S S M_{\text {min }}} \\
i & =1, \ldots, L ; j=1, \ldots, 20
\end{aligned}
$$

where $P S S M_{\text {max }}$ and $P S S M_{\text {min }}$ represent the maximal and minimal elements of PSSM.

\section{Multi-scale local average block}

We utilize Multi-scale Local Average Block (MLAB) algorithm to extract the conserved information of local regions. The original Average Block $(\mathrm{AB})$ algorithm was proposed by Jeong et al. [52]. Different from the original $A B$ algorithm, we use multi-scale size to split the matrix horizontally. The MLAB features can describe the local relationship between target residue and neighboring residues. Given a residue $R$, we denote $R^{-1}, R^{-2}, \ldots, R^{-5}$ be the five residues before $R$ in the sequence, and $R^{+1}, R^{+2}, \ldots, R^{+5}$ be the five residues after $R$ in the sequence. Then, $R^{ \pm 1}, R^{ \pm 2}, \ldots, R^{ \pm 5}$ are referred to as the ten sequential neighbors.

We split the information of target residue into six local sequential regions with varying composition, via global zone (A), bisection (B and C) and trichotomy (D, E and F). These local regions can describe multiple overlapping continuous and discontinuous interaction patterns, shown in Fig. 2. We calculate the mean of each local block as follows:

$$
L(k, j)=\frac{1}{B_{k}^{L}} \sum_{i=1}^{B_{k}^{L}} M_{k}^{L}(i, j) ; \quad k=1, \ldots, 6 ; j=1, \ldots, 20
$$

where $L(k, j)$ is the mean of $k$-th block in the column $j, B_{k}^{L}$ is the total number of rows in block $k$, and $M_{k}^{L}(i, j)$ is the value of cell in $i$-th row and $j$-th column of block $k$.

\section{Hexagon structure construction}

We build the hexagon structure for each target residue to describe its neighborhood information, as demonstrated in Fig. 3. We assume that $C_{\alpha}$ is the origin, $C_{\beta}$ is along the positive direction of $y$-axis, and $N$ is on the $x-y$ plane where $x$ is positive. The 3D space is partitioned along $y$ axis into six equal subspaces by three planes, and the angle between any two planes is $60^{\circ}$. Given a residue $R$, we locate nearest non-local $C_{\alpha}$ to $C_{\alpha}$ of residue $R$ within a certain distance in each subspace. Here, we say a residue is nonlocal to residue $R$ if and only if it is separated by at least three residues from residue $R$ in sequence. We call these six residues as spatial neighbors of residue $R$, denoted as $H_{R}^{1}, H_{R}^{2}, \ldots, H_{R}^{6}$.

We split the hexagon structure of target residue into six local spatial regions with varying composition, via global zone (A), bisection (B and C) and trichotomy (D, E and F). We calculate the mean of each local space as follows:

$$
H(k, j)=\frac{1}{B_{k}^{H}} \sum_{i=1}^{B_{k}^{H}} M_{k}^{H}(i, j) ; \quad k=1, \ldots, 6 ; j=1, \ldots, 20
$$

where $H(k, j)$ is the mean of $k$-th space in the column $j, B_{k}^{H}$ is the total number of rows in space $k$, and $M_{k}^{H}(i, j)$ is the value of cell in $i$-th row and $j$-th column of space $k$. 


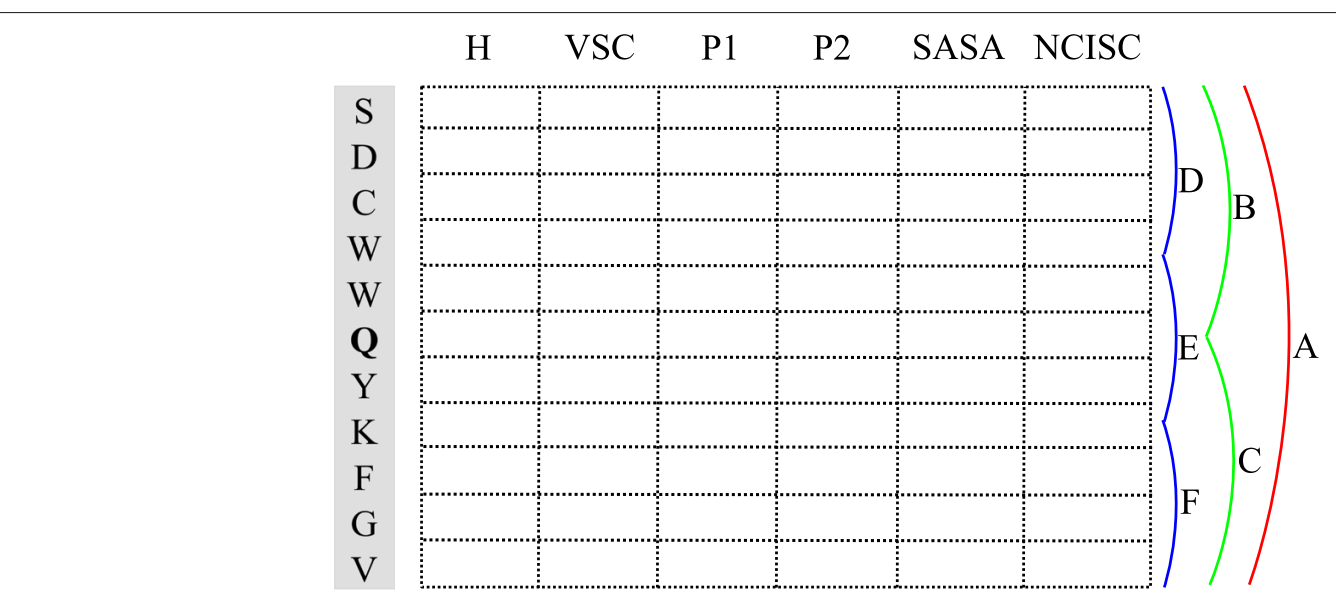

Fig. 2 Schematic diagram of Multi-scale Local Average Blocks feature extraction

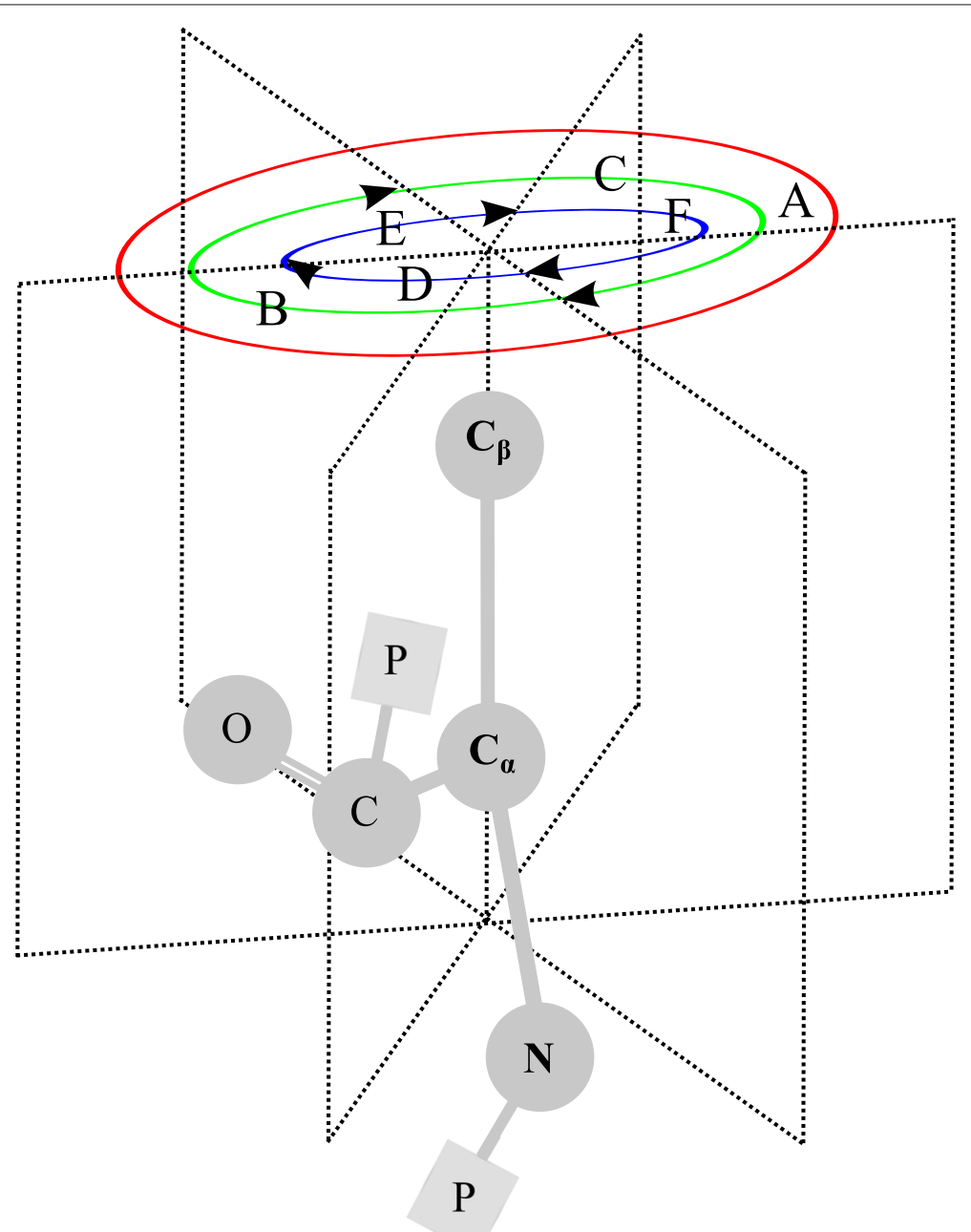

Fig. 3 Schematic diagram of Hexagon Structure Construction feature extraction 


\section{Extracting interface residues}

The above proposed features can be effectively applied to extract protein-protein interface residues and identify docking poses, as well as existing energy items. The energy items are listed as follows:

- amino acid contact energy - amino acid probabilities of interface residues [53].

- secondary structure contact energy - secondary structure probabilities of interface residues [53].

- structural neighborhood energy - probability of structural neighboring property on interface [54].

- dihedral angle energy - statistical analysis of dihedral angle correlation on interface [55].

- $\pi-\pi$ interaction energy - geometrical property on $\pi-\pi$ interaction [55].

- multi-scale local average block on protein 1D sequence.

- hexagon structure construction on protein 3D structure.

We use a trained support vector regression (SVR) model to rank docking poses, and then report the top-ranking poses with lowest energy values [56-58]. For the training set, we use $I_{r m s d}$ (rmsd value between predicted interfaces and native complexes) as the response values for all configurations of each pair of proteins, and the above energy items can be regarded as seven groups of features for each pose. Some configurations with the lowest predicted response values can be reported as the final result on the testing set. For a given pair of proteins, we use the trained SVR model to select top 10 predictions with lowest energy values.

\section{Results}

In this section, we compare our method to many existing methods for identifying protein-protein interfaces. Experiments show that our method performs better than some state-of-the-art methods on Benchmark v4.0 and the CAPRI targets, based on the prediction quality improved in terms of CAPRI evaluation criteria.

\section{Evaluation criteria}

A complex may contain several subunits and multiple binding interfaces. Each binding interface in a complex occurs in a pair of subunits. Two residues between a pair of subunits are called interface residues, if any two atoms, one from each residue, interact. By interacting, the distance between two atoms from a pair of different residues is less than $6 \AA$.

According to CAPRI evaluation criteria [45], three evaluation measures are commonly used in protein-protein interface prediction. A pair of residues on different sides of interface is considered to be in contact if any of their atoms are within $6 \AA$. One is the fraction of native contacts $F_{n a t}$, defined as the number of correct residue-residue contacts in the predicted configuration divided by the number of contacts in the native complex. The other is the fraction of non-native contacts $F_{n o n-n a t}$, defined as the number of incorrect residues-residue contacts in the predicted configuration divided by the total number of contacts in that predicted pose. The third is rootmean-square deviation of interface $I_{r m s d}$, defined as rmsd value between all backbone atoms of interfaces in predicted pose and in native complex, after two interfaces are superimposed.

The CAPRI evaluation use different cutoffs on these three measures to assign predicted poses into four quality classes: Incorrect $\left(F_{\text {nat }}<10 \%\right.$ or $\left.I_{\text {rms }}>4.0 \AA\right)$, Acceptable $\left(10 \%<=F_{\text {nat }}<30 \%\right.$ and $\left.2.0 \AA<I_{\text {rmsd }}<=4.0 \AA\right)$, Medium $\left(30 \%<=F_{\text {nat }}<50 \%\right.$ and $1.0 \AA<I_{\text {rmsd }}<=$ $2.0 \AA)$, or High $\left(F_{n a t}>=50 \%\right.$ and $\left.I_{r m s d}<=1.0 \AA\right)$.

\section{Statistical analysis}

We analyze different regression models and evaluate the performance of energy items on CAPRI [45]. CAPRI is a community-wide experiment to assess the capacity of docking methods.

\section{Assessment of regression model}

To assess the effectiveness of regression model, we analyze the performance of Support Vector Regression [59] and Linear Regression [60] with same energy items on CAPRI, and the results are shown in Fig. 4. The average $I_{r m s d}$ value for cases by Support Vector Regression is $3.45 \AA$. The average $I_{r m s d}$ value for cases by Linear Regression is $3.57 \AA$. It confirms our hypothesis that Support Vector Regression can accurately identify the protein-protein interface.

\section{Assessment of energy items}

To assess the effectiveness of energy items, we analyze the performance of different cases on CAPRI. We reevaluate configurations selected by different energy items, and the results are shown in Fig. 5. The average $I_{\text {rmsd }}$ value for cases with sequence contact energy (amino acid contact energy, secondary structure contact energy) is $3.63 \AA$. The average $I_{r m s d}$ value for cases with structural interaction energy (structural neighborhood energy, dihedral angle energy, $\pi-\pi$ interaction energy) is $3.57 \AA$. The average $I_{r m s d}$ value for cases with multi-scale local energy (multi-scale local average block on protein 1D sequence, hexagon structure construction on protein 3D structure) is $3.51 \AA$. Average $I_{r m s d}$ values for these cases are less than that for cases with all energy items $(3.45 \AA)$. It confirms our hypothesis that the multi-scale local representations 


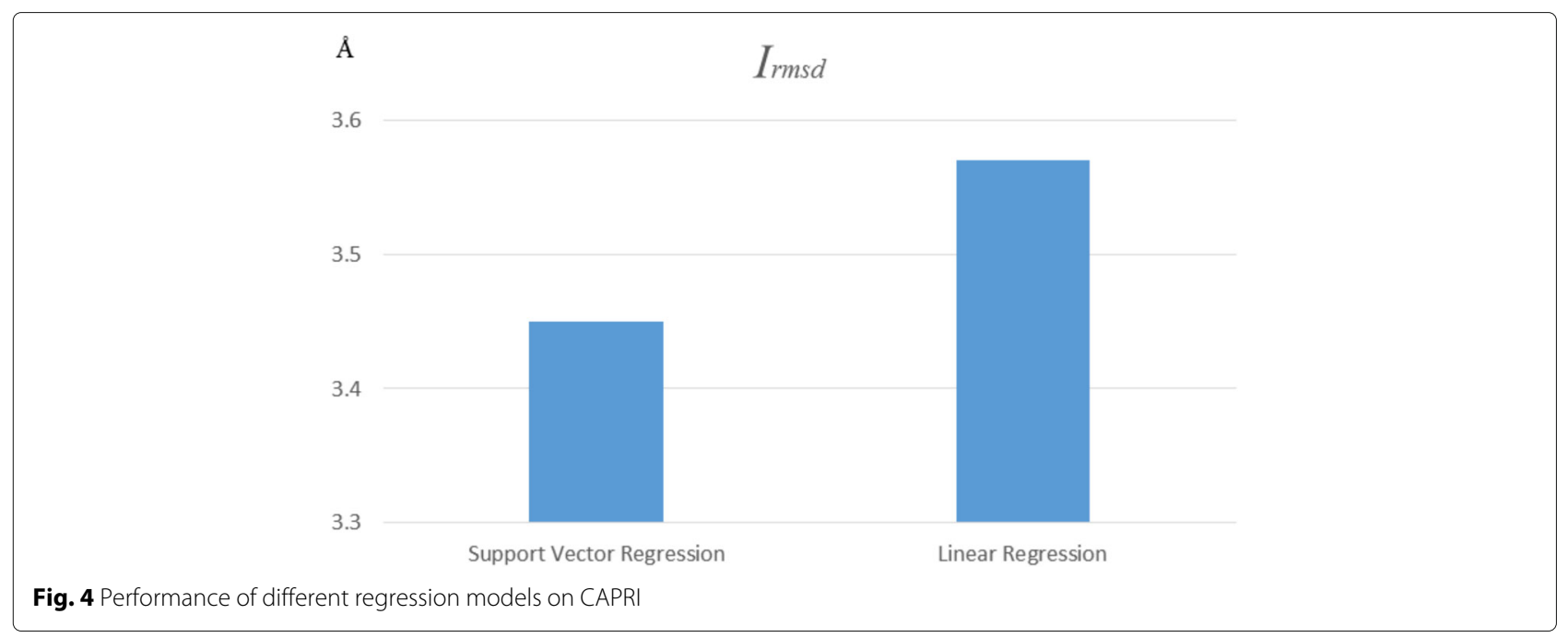

on sequence and structural information are the important factors to consider in the protein-protein interface prediction.

\section{Docking validation}

We evaluate the performance of our method on the protein-protein complexes in Benchmark 4.0 [46]. All targets in Benchmark 4.0 are classified into three categories: rigid-body (easy) cases, medium difficult cases and difficult cases, according to the magnitude of conformational change after binding. Our method is compared to SnapDock [38], InterEvDock [37] and FRODOCK 2.0 [36]. The success rate reports the percentage of cases for which at least one out of top 10 predictions is an acceptable or better solution on CAPRI criteria. The protein-protein docking results of different methods are shown in Table 2. The success rates by our method, FRODOCK 2.0, InterEvDock and SnapDock on Benchmark v4.0 are 41.5\%, 29.0\%, 29.4\% and $37.0 \%$, respectively. Our method improves the success rate at least by $4.5 \%$.

\section{Protein-protein interface prediction}

In this study, we compare our predicted interfaces with ZRANK [40, 41] and FiberDock(external tool) [28], and also with ClusPro [42]. We consider 79 complexes from Dockground [61] as the training set. In order to avoid over-fitting, we exclude complexes sharing more than $30 \%$ identity with cases in testing set. The average $I_{\text {rms }}$ value is $1.49 \AA$, and the overall $F_{n a t}$ and $F_{\text {non-nat }}$ values are $85 \%$ and $16 \%$.

\section{Evaluation on benchmark $v 4.0$}

On Benchmark v4.0, our method achieves average $I_{\text {rmsd }}$ value of $3.28 \AA$ and overall $F_{\text {nat }}$ value of $63 \%$, which

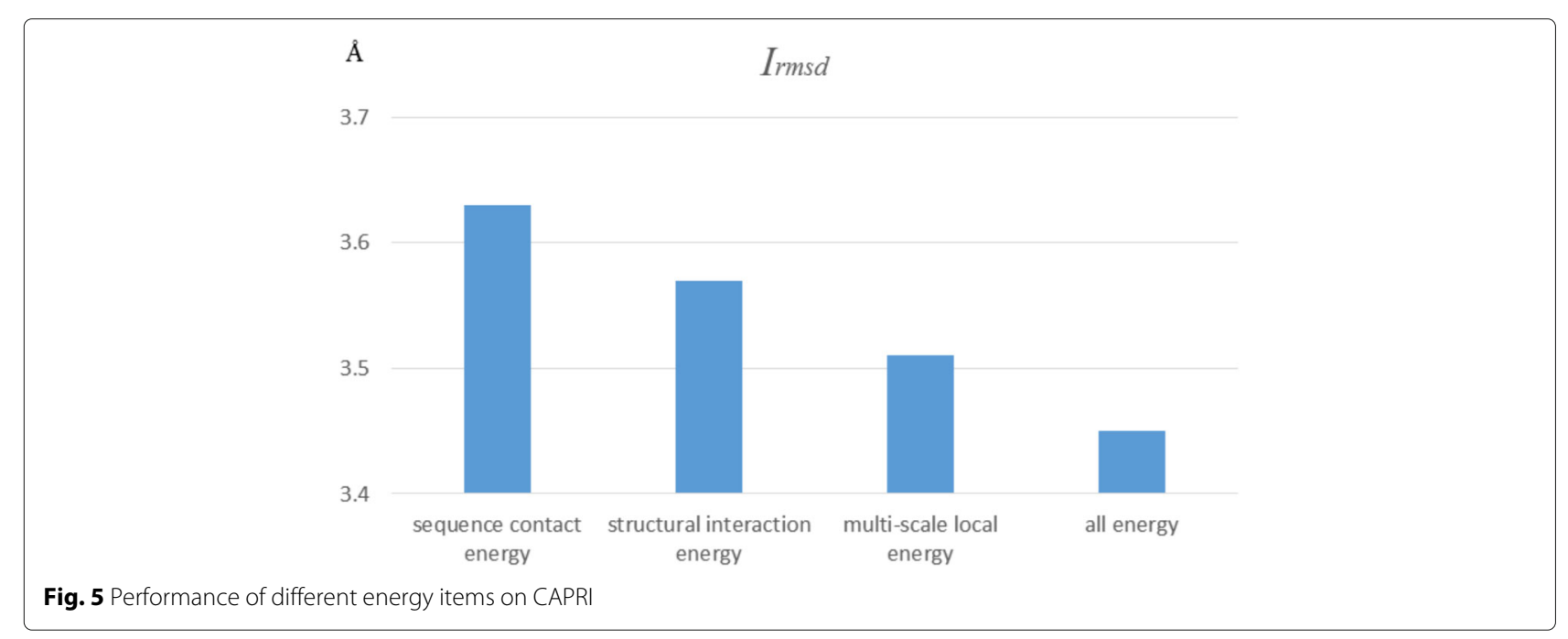


Table 2 The prediction results by our method, FRODOCK 2.0, InterEvDock and SnapDock on Benchmark v4.0

\begin{tabular}{ll}
\hline & success rate \\
\hline FRODOCK 2.0 & $29.0 \%(51 / 176)$ \\
InterEvDock & $29.4 \%(25 / 85)$ \\
SnapDock & $37.0 \%(57 / 154)$ \\
Our Method & $41.5 \%(73 / 176)$ \\
\hline
\end{tabular}

improves upon $I_{\text {rmsd }}$ of $3.89 \AA$ and $F_{\text {nat }}$ of $49 \%$ for ZRANK, and $I_{r m s d}$ of $3.99 \AA$ and $F_{\text {nat }}$ of $46 \%$ for ClusPro. Results are shown in Table 3. The complexes are classified into three categories, according to the magnitude of conformational change after binding. In rigid-body group, our method achieves average $I_{r m s d}$ value of $2.86 \AA$ and overall $F_{\text {nat }}$ value of $69 \%$, which improves upon $I_{\text {rmsd }}$ of $3.31 \AA$ and $F_{\text {nat }}$ of $56 \%$ for ZRANK, and $I_{\text {rmsd }}$ of $3.33 \AA$ and $F_{\text {nat }}$ of $55 \%$ for ClusPro. In medium difficulty group, our method achieves average $I_{\text {rmsd }}$ value of $3.35 \AA$ and overall $F_{\text {nat }}$ value of $59 \%$, which improves upon $I_{\text {rmsd }}$ of $4.46 \AA$ and $F_{\text {nat }}$ of $39 \%$ for ZRANK, and $I_{\text {rmsd }}$ of $4.71 \AA$ and $F_{\text {nat }}$ of $30 \%$ for ClusPro. In difficulty group, our method achieves average $I_{r m s d}$ value of $5.39 \AA$ and overall $F_{\text {nat }}$ value of $36 \%$, which improves upon $I_{\text {rmsd }}$ of $6.18 \AA$ and $F_{\text {nat }}$ of $28 \%$ for ZRANK, and $I_{\text {rmsd }}$ of $6.53 \AA$ and $F_{\text {nat }}$ of $21 \%$ for ClusPro.

\section{Evaluation on Capri}

We evaluate protein-protein interface prediction by our method, ZRANK and ClusPro on CAPRI. On 35 CAPRI targets, our method achieves average $I_{r m s d}$ value of $3.45 \AA$ and overall $F_{\text {nat }}$ value of $46 \%$, which improves upon $I_{\text {rms }}$ of $4.18 \AA$ and $F_{\text {nat }}$ of $40 \%$ for ZRANK, and $I_{\text {rmsd }}$ of $5.12 \AA$ and $F_{\text {nat }}$ of $32 \%$ for ClusPro. Our method predicts 9 incorrect, 12 acceptable, 12 medium, 2 high quality results. ZRANK+FiberDock predicts 14 incorrect, 7 acceptable, 7 medium, 7 high quality results. ClusPro predicts 13 incorrect, 11 acceptable, 8 medium, 3 high quality results.

\section{Binding sites identification}

Some existing methods use machine learning and statistical approaches to predict binding sites. Each comparison with an existing method is performed using the test data by the compared method in the literature.

\section{Comparison to metaPPI, meta-PPISP and pPI-Pred}

In this experiment, the test data in metaPPI [20] is used to predict binding sites. The data consists of 41 complexes, divided into two categories: enzyme-inhibitor (EI) and others. The overall $F_{\text {nat }}$ and $F_{\text {non-nat }}$ values for each prediction method are shown in Table 4 . The overall $F_{\text {nat }}$ values for our method, metaPPI, meta-PPISP and PPI-Pred achieve $62 \%, 28 \%, 38 \%$ and $38 \%$, respectively. The overall $F_{\text {non-nat }}$ values for these four methods achieve 34\%, $51 \%, 54 \%$ and $64 \%$, respectively. Our method improves the overall $F_{\text {nat }}$ value by at least $24 \%$. The average sizes of predicted interface residues for our method, metaPPI, meta-PPISP and PPI-Pred are 22.1, 13.2, 18.2 and 27.8, while the average size of actual interface residues is 22.7 . The number of residues predicted correctly for these four methods are 12.9, 5.5, 7.5 and 8.2.

\section{Comparison to proMate and pINUP}

Our method is compared to ProMate and PINUP. The test data is originally used by ProMate [16], including 57 unbound proteins and their complexes. The results are reported in Table 5 . The overall $F_{\text {nat }}$ values for our method, PINUP and ProMate achieve $60 \%, 42 \%$ and $13 \%$, respectively. The overall $F_{\text {non-nat }}$ values for these three methods achieve $45 \%, 55 \%$ and $47 \%$, respectively. Our method improves the overall $F_{\text {nat }}$ value by at least $19 \%$. The average sizes of predicted interface residues for our method, PINUP and ProMate are 25.6, 19.0 and 5.4, while the average size of actual interface residues is 22.6. The number of residues predicted correctly for these three methods are 12.6, 8.3 and 2.7.

\section{Case study}

We evaluate interface prediction of our method on two different cases.

\section{Interface prediction on sK/RR interaction}

We study HisKA domain of sensor histidine kinase (PF00512) and its partner response regulator domain (PF00072) in Pfam database [62]. Interface identification

Table 3 The prediction results by our method, ZRANK+FiberDock and ClusPro on Benchmark v4.0

\begin{tabular}{|c|c|c|c|c|c|c|c|c|c|c|}
\hline \multirow{2}{*}{ Subset ${ }^{a}$} & \multirow{2}{*}{ No. of cases } & \multicolumn{3}{|c|}{ Our Method } & \multicolumn{3}{|c|}{ ZRANK+FiberDock } & \multicolumn{3}{|c|}{ ClusPro } \\
\hline & & $\overline{I_{\text {rmsd }}}$ & $F_{\text {nat }}$ & $\overline{F_{\text {non-nat }}}$ & $\overline{I_{\text {rmsd }}}$ & $F_{\text {nat }}$ & $\overline{F_{\text {non-nat }}}$ & $I_{\text {rmsd }}$ & $F_{\text {nat }}$ & $\overline{F_{\text {non-nat }}}$ \\
\hline Rigid & 123 & 2.86 & $69 \%$ & $35 \%$ & 3.31 & $56 \%$ & $49 \%$ & 3.33 & $55 \%$ & $51 \%$ \\
\hline Medium & 29 & 3.35 & $59 \%$ & $39 \%$ & 4.46 & $39 \%$ & $59 \%$ & 4.71 & $30 \%$ & $69 \%$ \\
\hline Difficult & 24 & 5.39 & $36 \%$ & $58 \%$ & 6.18 & $28 \%$ & $67 \%$ & 6.53 & $21 \%$ & $77 \%$ \\
\hline Overall & 176 & 3.28 & $63 \%$ & $39 \%$ & 3.89 & $49 \%$ & $53 \%$ & 3.99 & $46 \%$ & $58 \%$ \\
\hline
\end{tabular}

a Subset is based on the magnitude of conformational change after binding 
Table 4 Comparison to metaPPI, meta-PPISP and PPI-Pred

\begin{tabular}{|c|c|c|c|c|c|c|c|c|}
\hline \multirow{2}{*}{ Type } & \multicolumn{2}{|c|}{ Our Method } & \multicolumn{2}{|c|}{ metaPPI } & \multicolumn{2}{|c|}{ meta-PPISP } & \multicolumn{2}{|c|}{ PPI-Pred } \\
\hline & $F_{\text {nat }}$ & $F_{\text {non-nat }}$ & $F_{\text {nat }}$ & $F_{\text {non-nat }}$ & $F_{\text {nat }}$ & $F_{\text {non-nat }}$ & $F_{\text {nat }}$ & $F_{\text {non-nat }}$ \\
\hline$E-1^{a}$ & $65 \%$ & $23 \%$ & $37 \%$ & $39 \%$ & $55 \%$ & $44 \%$ & $47 \%$ & $54 \%$ \\
\hline others & $59 \%$ & $42 \%$ & $22 \%$ & $59 \%$ & $26 \%$ & $61 \%$ & $31 \%$ & $71 \%$ \\
\hline Overall & $62 \%$ & $34 \%$ & $28 \%$ & $51 \%$ & $38 \%$ & $54 \%$ & $38 \%$ & $64 \%$ \\
\hline
\end{tabular}

${ }^{a} E-l$ is the type of enzyme-inhibitor

can be tested by using structural representatives of HisKA domain of SK (HK853; PDB ID code 2C2A chain A) and of RR domain (Spo0F; PDB ID code 1PEY chain A), as well as co-crystal structure of Spo0F in complex with $\mathrm{Spo0B}$ (PDB ID code $1 F 51$ chain A:E). We analyze 25 interacting residues, involving $13 \mathrm{SK}$ positions and $12 \mathrm{RR}$ positions. For HK853, predicted interface residues being part of interface are 267, 268, 271, 272, 275, 276, 291, 294 and 298 , as indicated by red boxes in Fig. 6. Predicted interface residues of SK belonging to non-interface are 245, 249, 253 and 256. For Spo0F, predicted interface residues being part of interface are 14, 15, 18, 19, 21 and 22, as indicated by red boxes in Fig. 6. Predicted interface residues of RR belonging to non-interface are $56,57,86,87,90$ and 91.

\section{Interface prediction on spirulina platensis}

We study spirulina platensis $\alpha$-subunit (PDB ID code $1 \mathrm{GHO}$ chain A) and $\beta$-subunit (PDB ID code $1 \mathrm{GHO}$ chain $\mathrm{B})$. We analyze 30 interacting residues, involving $15 \alpha$-subunit positions and $15 \beta$-subunit positions. For $\alpha$-subunit, predicted interface residues being part of interface are 5, 6, 9, 10, 24, 27, 31, 38 and 42, as indicated by red boxes in Fig. 7. Predicted interface residues of $\alpha$-subunit belonging to non-interface are 78, 79, 82, 83, 117 and 118 . For $\beta$-subunit, predicted interface residues being part of interface are $5,6,9,10,24,27,31,38$ and 42 , as indicated by red boxes in Fig. 7. Predicted interface residues of $\beta$ subunit belonging to non-interface are $78,79,82,83,117$ and 118 .

\section{Discussion}

Lots of protein-protein identification approaches are based on analyzing some different features, such as sequence and structural properties, as well as other physicochemical properties. Most of the features only describe the property of current interacting residues, but

Table 5 Comparison to PINUP and ProMate

\begin{tabular}{|c|c|c|c|c|c|c|}
\hline & \multicolumn{2}{|c|}{ Our Method } & \multicolumn{2}{|c|}{ PINUP } & \multicolumn{2}{|c|}{ ProMate } \\
\hline & $F_{\text {nat }}$ & $\overline{F_{\text {non-nat }}}$ & $F_{\text {nat }}$ & $F_{\text {non-nat }}$ & $F_{\text {nat }}$ & $\overline{F_{\text {non-nat }}}$ \\
\hline Overall & $60 \%$ & $45 \%$ & $42 \%$ & $55 \%$ & $13 \%$ & $47 \%$ \\
\hline
\end{tabular}

cannot represent real situation well, thus are insufficient to predict interface residues with high accuracy. Although many computational methods have been used to predict protein-protein interfaces, the effectiveness and robustness of previous prediction models can still be improved. Main improvements of our proposed method come from adopting the effective feature extraction models that can capture useful protein information. All results demonstrate that our method is a valuable technological tool for identifying protein-protein interface.

\section{Conclusions}

We identify two new features: multi-scale local average block and hexagon structure construction. Given a pair of proteins, we use the trained SVR model to select best poses. From experimental results, the prediction ability of our method is better than that of other existing state-of-the-art approaches. It demonstrates that our proposed method is a very promising and useful support tool for future proteomics research. In the future work,

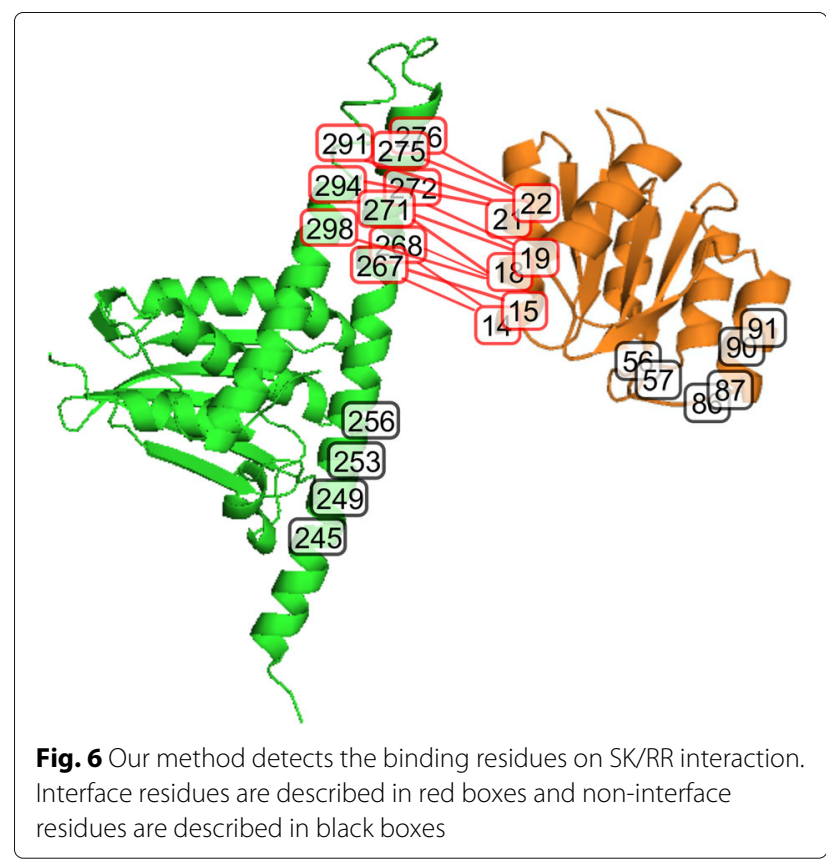




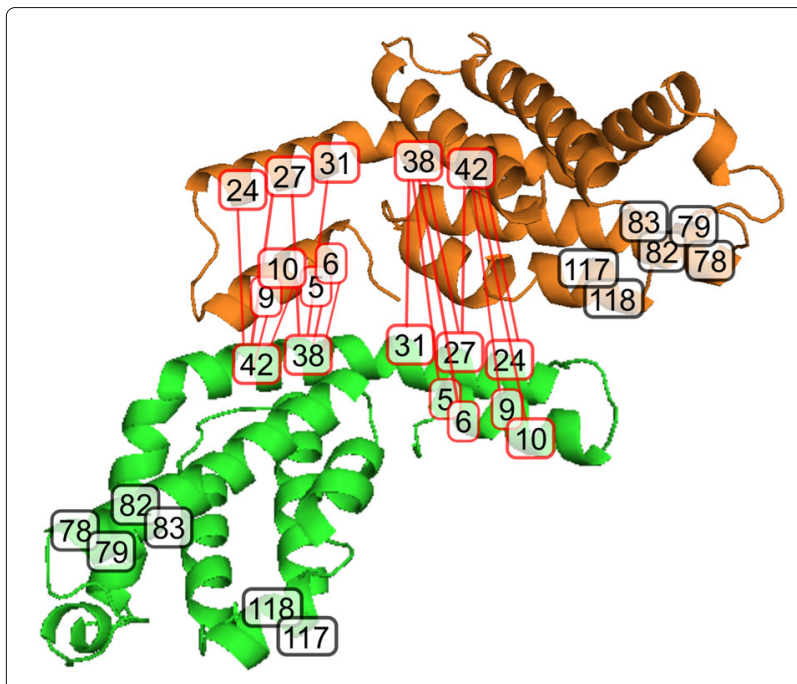

Fig. 7 Our method detects the binding residues on spirulina platensis. Interface residues are described in red boxes and non-interface residues are described in black boxes

we will extend our method to predict important special complexes.

\section{Abbreviations}

CAPRI: Critical Assessment of PRediction of Interactions; El: enzyme-inhibitor; H: Hydrophobicity; MLAB: Multi-scale Local Average Block; NCISC: Net Charge Index of Side Chains of amino acid; P1: Polarity; P2: Polarizability; PSSM: Position Specific Scoring Matrix; SASA: Solvent-Accessible Surface Area; SVR: Support Vector Regression; VSC: Volumes of Side Chains of amino acids

\section{Acknowledgments}

Authors would like to thank the reviewers for their helpful comments on the original manuscript. Authors are grateful to the conference committee of the 14th International Symposium on Bioinformatics Research and Applications (ISBRA 2018)

\section{About this supplement}

This article has been published as part of BMC Bioinformatics, Volume 20 Supplement 15, 2019: Selected articles from the 14th International Symposium on Bioinformatics Research and Applications (ISBRA-18): bioinformatics. The full contents of the supplement are available at https://bmcbioinformatics. biomedcentral.com/articles/supplements/volume-20-supplement- 15 .

\section{Authors' contributions}

Fei Guo conceived and designed the experiments; Dan Wang performed the experiments and analyzed the data; Quan Zou and Junhai Xu wrote the paper; Jijun Tang and Guang Yang reviewed the manuscript. All authors read and approved the final manuscript.

\section{Funding}

This research and this article's publication costs are supported by a grant from the National Science Foundation of China (NSFC 61772362).

\section{Availability of data and materials}

The datasets used or analysed during the current study are available from https://github.com/guofeieileen/binder.

\section{Ethics approval and consent to participate}

Not applicable.

\section{Consent for publication}

Not applicable.

\section{Competing interests}

The authors declare that they have no competing interests.

\section{Author details}

${ }^{1}$ College of Intelligence and Computing, Tianjin University, Tianjin, People's Republic of China. ${ }^{2}$ Institute of Fundamental and Frontier Sciences, University of Electronic Science and Technology of China, Chengdu, People's Republic of China. ${ }^{3}$ School of Economics, Nankai University, Tianjin, People's Republic of China. ${ }^{4}$ Department of Computer Science, City University of Hong Kong, Kowloon Tong, Hong Kong. ${ }^{5}$ Department of Computer Science and Engineering, University of South Carolina, Columbia, USA.

\section{Received: 14 August 2019 Accepted: 21 August 2019}

Published: 24 December 2019

\section{References}

1. Zhou H, Qin S. Interaction-site prediction for protein complexes: a critical assessment. Bioinformatics. 2007;23(17):2203-9.

2. Wass MN, David A, Sternberg MJE. Challenges for the prediction of macromolecular interactions. Curr Opin Struct Biol. 2011;21:382-90.

3. Pierce1 B, Wiehe $K$, Hwang $H$, Kim B, Vreven T, Weng Z. ZDOCK server: interactive docking prediction of protein-protein complexes and symmetric multimers. Bioinformatics. 2014;30(12):1771-3.

4. Torchala M, Moal I, Chaleil R, Fernandez-Recio J, Bates P. SwarmDock: a server for flexible protein-protein docking. Bioinformatics. 2013;29(6): $807-9$.

5. Jimenez-Garcia1 E, Pons C, Fernandez-Recio1 J. pyDockWEB: a web server for rigid-body protein-protein docking using electrostatics and desolvation scoring. Bioinformatics. 2013;29(13):1698-9.

6. Xu D, Si Y, Meroueh S. A computational investigation of small-molecule engagement of hot spots at protein-protein interaction interfaces. J Chem Inf Model. 2017;57:2250-72.

7. Krull L, Korff G, Elghobashi-Meinhardt N, Knapp E. ProPairs: a data set for protein-protein docking. Journal of Chemical Information and Modeling. 2015;55:1495-1507

8. Soni N, Madhusudhan MS. Computational modeling of protein assemblies. Curr Opin Struct Biol. 2017;44:179-89.

9. Rui MMB, Carreiras P, Simoes CJV, Silva CG. Enhancing scoring performance of docking-based virtual screening through machine learning. Curr Bioinforma. 2016;11(4):81-87.

10. Patel S, Tripathi R, Kumari V, Varadwaj P. Deepinteract: deep neural network based protein-protein interaction prediction tool. Curr Bioinforma. 2017;12(6):551-7.

11. Li BQ, Zhang YH, Jin ML, Huang T, Cai YD. Prediction of protein-peptide interactions with a nearest neighbor algorithm. Curr Bioinforma. 2018;13(1):14-24.

12. Wei L, Liao M, Gao X, Zou Q. An improved protein structural prediction method by incorporating both sequence and structure information. IEEE Trans Nanobioscience. 2015;14(4):339-49.

13. Zeng J, Li D, Wu Y, Zou Q, Liu X. An empirical study of features fusion techniques for protein-protein interaction prediction. Curr Bioinforma. 2016;11(1):4-12.

14. Wei L, Xing P, Zeng J, Chen J, Su R, Guo F. Improved prediction of protein-protein interactions using novel negative samples, features, and an ensemble classifier. Artif Intell Med. 2017;83:67-74.

15. Wei L, Tang J, Zou Q. Local-DPP: an improved DNA-binding protein prediction method by exploring local evolutionary information. Inf Sci. 2017;384:135-44.

16. Neuvirth H, Raz R, Schreiber G. Promate: a structure based prediction program to identify the location of protein-protein binding sites. J Mol Biol. 2004;338:181-99.

17. Bradford JR, Westhead DR. Improved prediction of protein-protein binding sites using a support vector machines approach. Bioinformatics. 2005;21 (8):1487-94.

18. Liang S, Zhang C, Liu S, Zhou Y. Protein binding site prediction using an empirical scoring function. Nucleic Acids Res. 2006;34(13):3698-707.

19. Qin S, Zhou HX. meta-ppisp: a meta web server for protein-protein interaction site prediction. Bioinformatics. 2007;23(24):3386-7.

20. Huang B, Schröder M. Using protein binding site prediction to improve protein docking. Gene. 2008;422:14-21.

21. Konc J, Janežič D. Probis algorithm for detection of structurally similar protein binding sites by local structural alignment. Bioinformatics. 2010;26(9):1160-8 
22. Konc J, Janežič D. Probis: a web server for detection of structurally similar protein binding sites. Nucleic Acids Res. 2010;38:W436-W440.

23. Heifetz A, Katchalski-Katzir E, Eisenstein M. Electrostatics in protein-protein docking. Protein J. 2002;11(3):571-87.

24. Schneidman-Duhovny D, Inbar Y, Nussinov R, Wolfson HJ. Geometry-based flexible and symmetric protein docking. Proteins. 2005;60(2):224-31

25. Fernández-Recio J, Totrov M, Skorodumov C, Abagyan R. Optimal docking area: A new method for predicting protein-protein interaction sites. Proteins. 2005;58(1):134-43.

26. Schneidman-Duhovny D, Inbar Y, Nussinov R, Wolfson HJ. PatchDock and SymmDock: servers for rigid and symmetric docking. Nucleic Acids Res. 2005;33:363-7.

27. Schneidman-Duhovny D, Nussinov R, Wolfson HJ. Automatic prediction of protein interactions with large scale motion. Proteins. 2007;69:764-73.

28. Mashiach E, Nussinov R, Wolfson HJ. FiberDock: flexible induced-fit backbone refinement in molecular docking. Proteins. 2009;78(6): 1503-19.

29. Ma B, Elkayam T, Wolfson H, Nussinov R. Protein-protein interactions: structurally conserved residues distinguish between binding sites and exposed protein surfaces. Proc Natl Acad Sci. 2003;100(10):5772-7.

30. Xu D, Tsai CJ, Nussinov R. Hydrogen bonds and salt bridges across protein-protein interfaces. Protein Eng. 1997;10(9):999-1012.

31. Ansari $\mathrm{S}$, Helms V. Statistical analysis of predominantly transient protein-protein interfaces. J Comput Chem. 2005;61(2):344-55.

32. Cho K, Kim D, Lee D. A feature-based approach to modeling protein-protein interaction hot spots. Nucleic Acids Res. 2009;37(8): 2672-87.

33. Brooks BR, Bruccoleri RE, Olafson BD, States DJ, Swaminathan S, Karplus M. CHARMM: a program for macromolecular energy, minimization, and dynamics calculations. J Comput Chem. 1983;4(2):187-217.

34. Wang J, Wolf RM, Caldwell JW, Kollman PA, Case DA. Development and testing of a general AMBER force field. J Comput Chem. 2004;25:1157-74.

35. Lindahl E, Hess B, Spoel D. GROMACS 3.0: a package for molecular simulation and trajectory analysis. J Mol Model. 2001;7(8):306-17.

36. Ramrez-Aportela E, Lopez-Blanco J, Chacon P. FRODOCK 2.0: fast protein-protein docking server. Bioinformatics. 2016;32:2386-8.

37. Yu J, Vavrusa M, Andreani J, Rey J, Tuffery P, Guerois R. InterEvDock: a docking server to predict the structure of protein-protein interactions using evolutionary information. Nucleic Acids Res. 2016;44:W542-W549.

38. Estrin M, Wolfson H. SnapDock: template-based docking by Geometric Hashing. Bioinformatics. 2017;33:i30-i36.

39. Nadalin F, Carbone A. Protein-protein interaction specificity is captured by contact preferences and interface composition. Bioinformatics. 2018:34:459-68.

40. Pierce $B$, Weng Z. A combination of rescoring and refinement significantly improves protein docking performance. Proteins. 2008;72(1):270-9.

41. Vreven $\mathrm{T}$, Hwang $\mathrm{H}$, Weng Z. Integrating atom-based and residue-based scoring functions for protein-protein docking. Proteins. 2011;20(9):1576-86.

42. Comeau SR, Gatchell DW, Vajda S, Camacho CJ. ClusPro: an automated docking and discrimination method for the prediction of protein complexes. Bioinformatics. 2004;20(1):45-50.

43. Schueler-Furman O, Wang C, Baker D. Progress in protein-protein docking: atomic resolution predictions in the CAPRI experiment using RosettaDock with an improved treatment of side-chain flexibility. Proteins. 2005:60:187-94.

44. Dominguez C, Boelens R, Bonvin A. HADDOCK: a protein-protein docking approach based on biochemical or biophysical information. J Am Chem Soc. 2003;125:1731-7.

45. Janin J, Henrick K, Moult J, Eyck LT, Sternberg M, Vajda S, Vakser I, Wodak S. CAPRI: A critical assessment of predicted interactions. Proteins. 2003;52(1):2-9.

46. Hwang $\mathrm{H}$, Vreven $\mathrm{T}$, Janin J, Weng Z. Protein-protein docking benchmark version 4.0. Proteins. 2010;78:3111-4.

47. Guo F, Li SC, Wang L, Zhu D. Protein-protein binding site identification by enumerating the configurations. BMC Bioinformatics. 2012;13:158.

48. Ding Y, Tang J, Guo F. Predicting protein-protein interactions via multivariate mutual information of protein sequences. BMC Bioinformatics. 2016;17:389-410.
49. Ding Y, Tang J, Guo F. Identification of protein-protein interactions via a novel matrix-based sequence representation model with amino acid contact information. Int J Mol Sci. 2016;17:1623.

50. Altschul SF, Gish W, Miller W, Myers EW, Lipman DJ. Basic local alignment search tool. J Mol Biol. 1990;215(3):403-10.

51. Henikoff S, Henikoff JG. Amino acid substitution matrices from protein blocks. Proc Natl Acad Sci. 1091;89(22):5-9.

52. Jeong JC, Lin X, Chen XW. On position-specific scoring matrix for protein function prediction. IEEE/ACM Trans Comput Biol Bioinforma. 2011;8:308-15.

53. Guo F, Li SC, Fan Y, Wang L. Identifying protein-protein binding sites with a combined energy function. Current Protein Pept Sci. 2014;15(6): 540-52

54. Guo F, Li SC, Wei Z, Zhu D, Shen C, Wang L. Structural neighboring property for identifying protein-protein binding sites. BMC System Biology. 2015;9(Suppl 5:S3). https://www.ncbi.nlm.nih.gov/pmc/articles/ PMC4565107/.

55. Guo F, Li SC, Du P, Wang L. Probabilistic models for capturing more physicochemical properties on protein-protein interface. J Chem Inf Model. 2014;54(6):1798-809.

56. Guo F, Li SC, Ma W, Wang L. Detecting protein conformational changes in interactions via scaling known Structures. J Comput Biol. 2013;20(10): 765-79.

57. Guo F, Ding Y, Li SC, Shen C, Wang L. Protein-protein interface prediction based on hexagon structure similarity. Comput Biol Chem. 2016;63:83-88.

58. Guo F, Ding Y, Li Z, Tang J. Identification of protein-protein interactions by detecting correlated mutation at the interface. J Chem Inf Model. 2015;55(9):2042-9.

59. Drucker H, Burges C, Kaufman L, Smola A, Vapnik V. Support Vector Regression Machines. Adv Neural Inf Process Syst. 1997;9:155-61.

60. Yan X. Linear regression analysis: theory and computing. World Sci. 2009 https://dl.acm.org/citation.cfm?id=1717831. http://www.manalhelal. com/Books/geo/LinearRegressionAnalysisTheoryandComputing.pdf.

61. Liu S, Gao Y, Vakser I. Dockground protein-protein docking decoy set. Bioinformatics. 2008;24:2634-5.

62. Finn RD, Bateman A, Clements J, Coggill P, Eberhardt RY, Eddy SR, Heger A, Hetherington K, Holm L, Mistry J, Sonnhammer EL, Tate J, Punta M. The Pfam protein families database. Nucleic Acids Res. 2007;36: D281-D288.

\section{Publisher's Note}

Springer Nature remains neutral with regard to jurisdictional claims in published maps and institutional affiliations.

\section{Ready to submit your research? Choose BMC and benefit from}

- fast, convenient online submission

- thorough peer review by experienced researchers in your field

- rapid publication on acceptance

- support for research data, including large and complex data types

- gold Open Access which fosters wider collaboration and increased citations

- maximum visibility for your research: over $100 \mathrm{M}$ website views per year

At BMC, research is always in progress.

Learn more biomedcentral.com/submissions 